time of presenting complaints. Ethics approval was granted by Columbia University.

Results: Data was collected for a two-week period approximately three weeks after the hurricane made landfall. The FMS saw 2,154 patients over a 14-day period. The population of patients (median age $=43$ years [IQR 39 years $]$ ) assessed was bimodal in distribution, with one peak in children at 1 year. A second peak occurred at age 53 years. $60.2 \%$ of presenting complaints were infection- or chronic disease-related. Musculoskeletal complaints were the third most common. Chi-squared tests revealed no statistically significant change in the frequency of specific types of complaints between the start and end of data collection.

Discussion: In the weeks after Hurricane Maria, infants and elderly were seen to predominantly seek medical care. Likely related to the collapse of the healthcare infrastructure, there was a high prevalence of infection-related and chronic medical conditions. The data support the need to focus resources to treat vulnerable populations, infectious issues, and chronic medical conditions. Prehosp Disaster Med 2019;34(Suppl. 1):s114-s115 doi:10.1017/S1049023X19002437

\section{Description of Disaster Forms Usage in Health Cluster in Lombok and Palu Earthquake}

Miss Madelina Ariani, Mrs. Bella Donna

Center For Health Policy and Management, Faculty of Medicine, Public Health, and Nursing, Universitas Gadjah Mada, Yogyakarta, Indonesia

Introduction: The effort of medical and health services distribution requires data. However, the data and information were ignored in an emergency situation. For improving the distribution of data and information, the Center of Health Policy and Management, Faculty of Medicine, Public Health, and Nursing Universitas Gadjah Mada (UGM) developed forms based on Health Crisis Response Guideline by Ministry of Health 2016 and the World Health Organization (WHO).

Aim: Describing the implementation and development of forms based on Lombok and Central Sulawesi earthquake in 2018 for health cluster.

Methods: The form contains (1) a volunteer registration form; (2) a monitoring potential outbreak disease form; (3) health problem in health cluster daily report form; (4) a chronological situation form. This will be implemented in health policymaking by the Sulawesi district health office (DHO) and will be regularly analyzed in every week.

Results: North Lombok DHO, Central Sulawesi health office, and volunteers accepted these forms well. Periodically volunteers had reported their activity to DHO. All these reports contain many health indicators including environmental health. Reproductive health and health promotion. Implementation of this form in the other type of disaster in Indonesia is suggested.

Discussion: First, these forms are important to attach to the guideline of health crisis response in order to be accessed by all DHO. Second, all forms are printed documents. It needs to develop into data input and analysis applications.

Prehosp Disaster Med 2019;34(Suppl. 1):s115

doi:10.1017/S1049023X19002449
Deuce and a Half with a Twist: Repurposing Old Technology to Save Lives in Swiftwater Rescue during Urban and Small Stream Flash Flooding Dr. Sonia Jaslow ${ }^{1}$, Dr. David Jaslow ${ }^{1}$, Mr. Derek Zecher ${ }^{1}$, Mr. Ryan Synnestvedt ${ }^{1}, M r$. Nathaniel Zabustecher ${ }^{1}$, Mr. Kevan Melly ${ }^{3}$, Dr. Ryan Overberger ${ }^{2}$

1. Bryn Athyn Fire Company, Jenkintown, United States

2. Einstein Medical Center, Philadelphia, United States

3. Huntingdon Valley Fire Company, Huntingdon Valley, United States

Introduction: Vehicles stranded in rising water account for the majority of swiftwater rescues (SWR) during urban and small stream flash flooding. Multiple simultaneous SWR incidents are commonplace during severe storms. Historically, SWR teams have pursued a "reach, throw, row, go" strategy. However, reach and throw attempts are usually futile. Boat operations and/or in-water rescue attempts can be technically complicated, time-consuming, and a drain on rescuer resources. Aim: To design an ideal SWR modality for use during urban and small stream roadway flooding.

Methods: SWR objectives, strategy, and tactics were mapped against various transportation modalities to develop the safest solution for urban and small stream flood response.

Results: High water vehicles (HWV), such as the "deuce and a half" $6 \times 6$ military truck, represent a new standard for SWR practicality and safety as they can reduce rescuer time in-water. HWVs are heavy and high enough to be stable on roadways in most flash flooding conditions. A properly designed emergency response package includes a fording kit, multi-directional floodlights for nighttime operations, public safety radios, and a siren that doubles as a public address system to coach victims as a rescue is initiated. Deployable ladders enable rescuer egress from and victim access to a covered lighted cargo bed that holds PPE, throw bags, and rescue rings; a deployable "boat in a bag" for victims who require ferrying; and a heated seating area where medical evaluation can be conducted while staying dry.

Discussion: SWRs are dangerous resource-intensive incidents which account for more rescuer morbidity/mortality than all other technical rescue sub-types combined. These incidents will increase in frequency and severity worldwide due to climate change and overdevelopment. If rescue conditions are still tenable, HWVs are the most efficient and effective platform for conducting SWR from flooded roadways while decreasing safety risks to first responders and victims.

Prehosp Disaster Med 2019;34(Suppl. 1):s115

doi:10.1017/S1049023X19002450

\section{Developing a Knowledge Program for Large Scale Prehospital Assistance During Disasters and Big Incidents Dr. Will Van Roessel, Dr. Carian Cools \\ IFV, the National Institute of Safety, Netherlands}

Introduction: In the Netherlands, we started in 2016 with a new procedure for large scale medical assistance during a crisis. The normal daily assistance in the Netherlands is organized on a regional level, and we have 25 regions. These regions are far too small to handle big incidents, and cooperation is needed on a 
higher level to generate enough capacity. However, the Aim is that most emergency workers continue to do their own work in standard procedures, we also need more coordination, information management, transition of "stay and play" to "scoop and run" and deploying volunteers and citizens.

Aim: We developed the model practice-based, however, we have little big incidents. We feel the urgency to compare this practice to international knowledge.

Methods: The goal is twofold: validation of the starting points of our model, but also further improvement: speeding up the transport and treatment of patients, improvement of capacity, safety of the ambulance staff - especially with terrorist attacks or contamination, civil participation. We held the first survey on scientific literature in English, related to items in our prehospital assistance model. (the article is not yet published).

Results: The conclusion was, that scientific articles are rare, however, a lot of information is given about the practical course of incidents. Scientific research to explore these experiences is rare, partly due to a missing universal terminology on disaster medicine.

Discussion: We want to contribute to enlarging the scientific knowledge on large scale prehospital assistance. We expect that a lot of practical experience can be unlocked by bringing together experts in this field. We want to present the Dutch model, with a focus and invitation to compare this with the models in other countries, to compare experiences, to deepen them and to stimulate international research. We want to commit ourselves to facilitate this.

Prehosp Disaster Med 2019;34(Suppl. 1):s115-s116

doi:10.1017/S1049023X19002462

\section{Developing a Public Health Risk Assessment Toolkit for Mass Gatherings and Trialling for an International Multi-Sport Mass Gathering Event \\ Ms. Ellen Bloomer ${ }^{1}$, Tina Endericks ${ }^{1,2}$}

1. Public Health England, London, United Kingdom

2. WHO Collaborating Centre on Mass Gatherings and Global Health Security, London, United Kingdom

Introduction: Risk assessment for mass gatherings (MGs) is undertaken to enable public health authorities to systematically identify and assess the generic characteristics of an MG, which introduce or enhance particular threats and develop measures to reduce or mitigate these threats. The World Health Organization Collaborating Centres on Mass Gatherings and Global Health Security (WHO CCs) produced a comprehensive guide to MGs called "Public Health for Mass Gatherings: Key Considerations" (KC2). This is being converted into an eLearning resource. A public health risk assessment toolkit is being developed by the WHO CCs to complement and guide organizers in their planning process for the health risks associated with an MG event. Preparations for the Birmingham 2022 Commonwealth Games (BCG) are underway and it is important to involve a public health element in the planning for the BCG.

Aim: To develop a public health risk assessment toolkit for MGs and pilot it as part of the planning process for the BCG.
Methods: Based on $\mathrm{KC} 2$ principles, methods included developing and finalizing a public health risk assessment toolkit for MGs. This study also piloted the toolkit for the BCG.

Results: A toolkit will be developed. Key learning will be documented on how the toolkit can be improved. The pilot will identify the key public health risks for the BCG, and assess how to mitigate them.

Discussion: The development of this toolkit will be an innovative contribution to the resources available for those organizing MGs. It will support organizers to conduct risk assessments and thus maximize the potential for health from the event. Piloting the toolkit for the BCG is an opportunity for validating it, and provides valuable learning for its use in future events. It will support the risk assessment process for the BCG and share learning regarding the key risks for this event.

Prehosp Disaster Med 2019;34(Suppl. 1):s116

doi:10.1017/S1049023X19002474

\section{Developing an Educational Strategy for Delivering an E-learning Disaster Medicine Course for Undergraduate Students in US Medical Schools}

Dr. Michael Molloy ${ }^{1,2}$, Edmond Power ${ }^{2}$, A Prof. Gregory

R. Ciottone ${ }^{1,3}$

1. BIDMC Fellowship In Disaster Medicine, Boston, United States

2. University College Dublin, Belfield, Dublin, Ireland

3. Department of Emergency Medicine, Harvard Medical School, Boston, United States

Introduction: Disaster Medicine (DM) is a discipline arising from the marriage of emergency medicine and disaster management. The importance of DM has recently increased, with current wildfire situations throughout the world being examples of mass scale disasters with significant human morbidity and mortality. DM deals with preparedness, mitigation, response, recovery, and prevention of disasters (1).

Aim: To develop an educational strategy and reusable format for delivering undergraduate DM courses online. Man-made, weather-related, humanitarian, and technological disasters occur all around the globe annually, yet the majority of medical schools do not have an undergraduate DM program. This project developed an online course structure accessible to medical schools and students throughout the world.

Methods: Learning theories and models of learning were used to construct a course layout that encouraged students to be active learners, developed long-term retention strategies, and facilitated assessment for and of learning. This was accomplished through innovative educational modalities, including novel apps and external online resources. The course focuses heavily on outcome-based education with an emphasis on the development of applicable skills. Each lecture is divided into a series of learning objectives to allow students to master concepts sequentially, followed by questions to make use of the "testing effect" (2).

Results: Focused review of current medical education literature reveals that students learn best when given short, outcomefocused "mini-lectures" followed by low-stakes assessment and feedback. 